\title{
Flebotomíneos coletados em matas remanescentes e abrigos de animais silvestres de zoológico no perímetro urbano de Maringá, sul do Brasil. Estudo preliminar
}

\author{
Phlebotomines collected in remaining florests and wild animal \\ shelters in zoological garden in the urban area \\ of Maringá, Paraná State, shoutern Brazil. \\ Preliminary study \\ Ueslei Teodoro, João Balduíno Kühl, Mauro Rodrigues, Élcio Silvestre dos \\ Santos, Demilson Rodrigues dos Santos e Lídia Maria da Fonseca Maróstica
}

\begin{abstract}
Resumo Um caso de leishmaniose tegumentar com provável infecção em uma das áreas de matas remanescentes no perímetro urbano de Maringá, Paraná, Brasil, o desconhecimento da fauna e do comportamento de flebotomíneos nestas matas despertaram o interesse desta investigação. Os flebotomíneos foram coletados com armadilhas de Falcão instaladas em matas remanescentes do Parque do Ingá, Bosque Dois e Horto Florestal, de junho a setembro de 1995, no período noturno. Nestas áreas coletaram-se 3.532 flebotomíneos, prevalecendo Lutzomyia (Nyssomyia) whitmani (Antunes \& Coutinho, 1939) com 3.395 (96,1\%) exemplares. No Parque do Ingá, onde as coletas também foram feitas em abrigos de animais silvestres, mantidos em zoológico, foram coletados 2.907 flebotomíneos, dos quais 1.723 nestes abrigos. Os resultados mostram melhor adaptação de Lutzomyia whitmani nas matas remanescentes no perímetro urbano de Maringá e sua freqüência nos abrigos dos animais silvestres mantidos em cativeiro.
\end{abstract}

Palavras-chaves: Leishmaniose urbana. Flebotomíneos vetores. Lutzomyia whitmani.

\begin{abstract}
The present study was undertaken after the detection of one case of cutaneous leishmaniasis with presumed infection in one of the three remaining wooded areas in the urban area of the city of Maringá, Southern Brazil; also in view of the lack of knowledge about sand flies and their behavior. From June to September, 1995, sand flies were caught with Falcão traps during the night in the remaining wooded areas (Parque do Ingá, Bosque Dois and Horto Florestal). A total of 2,907 sand flies were caugth in Parque do Ingá; 1,723 of them were aught in forest traps and 1,184 in wild animal shelter traps at the zoo. The results show that Lutzomyia whitmani is better adapted to the three areas under study and that it frequently occurs in wild animal shelters within the urban perimeter of Maringá.
\end{abstract}

Key-words: Urban leishmaniasis. Sand flies. Lutzomyia whitmani.

Departamento de Análises Clínicas da Universidade Estadual de Maringá, Maringá, PR.

Financiado pela Fundação Nacional de Saúde, Ministério da Saúde - Diretoria Regional do Estado do Paraná.

Endereço para correspondência: Dr. Ueslei Teodoro. Depto. de Análises Clínicas/UEM. Av. Colombo 5.790, 87020-900 Maringá, PR, Brasil.

Recebido para publicação em 29/10/97. 
Os relatos de capturas de flebotomíneos em áreas urbanas no Brasil têm sido freqüentes 51316 demonstrando a capacidade de algumas espécies se adaptarem aos ambientes, por vezes, acentuadamente antropogênicos. Casos humanos e caninos de leishmaniose tegumentar e leishmaniose visceral têm sido notificados em áreas urbanas de Belo Horizonte, Rio de Janeiro, São Luís e Teresina16, tornando-se evidente a urbanização destas doenças que aguardam por medidas que venham a auxiliar o seu controle21. Cabe lembrar que de 1980 a 1996 mais de 300 mil casos de leishmanioses foram notificados no Brasil8 911.

A notificação de leishmaniose tegumentar no Estado do Paraná vem abrangendo diversos municípios 15 . Um caso de leishmaniose tegumentar em um vigilante noturno foi notificado em 1995 no município de Maringá, pela Secretária Municipal de Saúde. O local de trabalho do referido vigilante é uma área de lazer com um pequeno zoológico (Parque do Ingá), no perímetro urbano deste município.

Os fatos supracitados motivaram a realização de estudos da fauna e freqüência de flebotomíneos em abrigos de animais silvestres mantidos em cativeiro, na mata do Parque do Ingá, e em duas outras áreas com cobertura vegetal primitiva, todas dentro do perímetro urbano de Maringá.

\section{MATERIAL E MÉTODOS}

Descrição da área. As áreas onde foi realizada esta investigação pertencem ao município de Maringá, situado no noroeste do Paraná a $23^{\circ} 25^{\prime}$ de latitude sul e $51^{\circ} 25^{\prime}$ de longitude Oeste de Greenwich. A precipitação média anual fica entre 1.500 e $1.600 \mathrm{~mm}$. No trimestre menos chuvoso (junho, julho e agosto) as precipitações constatadas foram de 200 a $250 \mathrm{~mm}$ e no mais chuvoso (dezembro, janeiro e fevereiro) de 500 a $550 \mathrm{~mm}$. As temperaturas médias anuais ficam entre $20-21^{\circ} \mathrm{C}$, com médias máximas de 27 a $28^{\circ}$ e médias mínimas entre 16 e $17^{\circ} \mathrm{C}$.

O Parque do Ingá, o Bosque Dois e o Horto Florestal, onde foi efetuada a pesquisa, são áreas de preservação cobertas com matas primitivas modificadas, localizadas no perímetro urbano do município de Maringá (Figura 1).

Coletas de flebotomíneos. As coletas de flebotomíneos foram feitas com armadilhas de Falcão4. No Parque do Ingá, as armadilhas foram

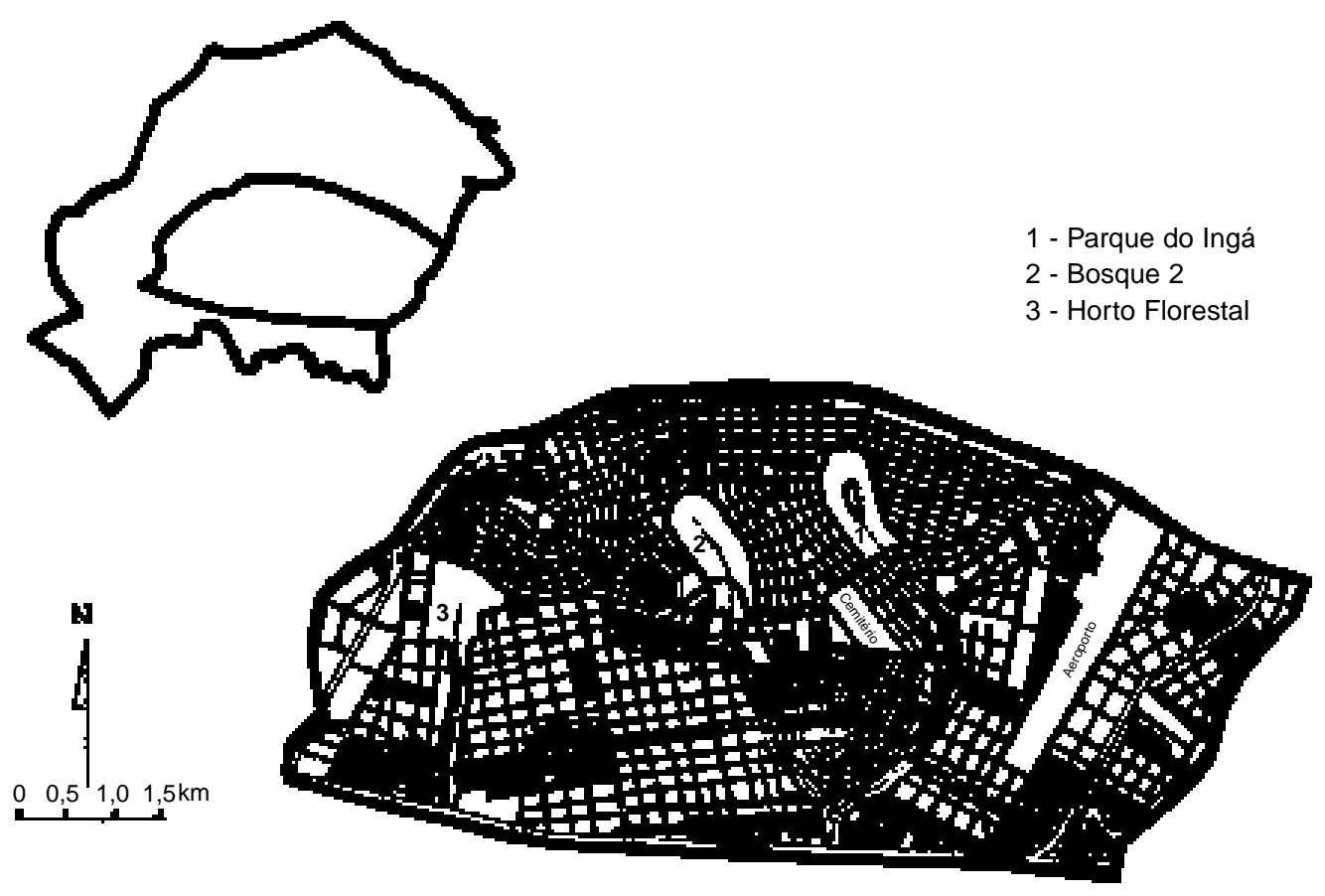

Figura 1 - Localização das áreas de pesquisa no perímetro urbano de Maringá, Paraná, Brasil. 
instaladas nos abrigos de queixadas \{Tayassu pecari (Link, 1795)\}, macacos \{Cebus apella (Linnaeus, 1758)\}, aves de diversas espécies, e no abrigo de uma onça P Panthera onca (Linnaeus, 1758)\}(Figura 2). As coletas foram feitas em: a) 12 noites consecutivas em junho de 1995, das 18 às 21 horas, num total de 36 horas; b) 5 noites seguidas em julho das 21 às 24 horas, somando 15 horas; 3 noites consecutivas das 0 às 3 horas, totalizando 9 horas; 2 noites seguidas das 3 às 6 horas, dando 6 horas. No total foram 22 noites e 66 horas de coletas. Concomitantemente, nas 22 noites 4 armadilhas foram instaladas nas partes mais internas da mata, nas proximidades dos abrigos de animais silvestres. Em 3 noites seguidas, duas em agosto e uma em setembro, das 19 às 22 horas, 8 armadilhas foram

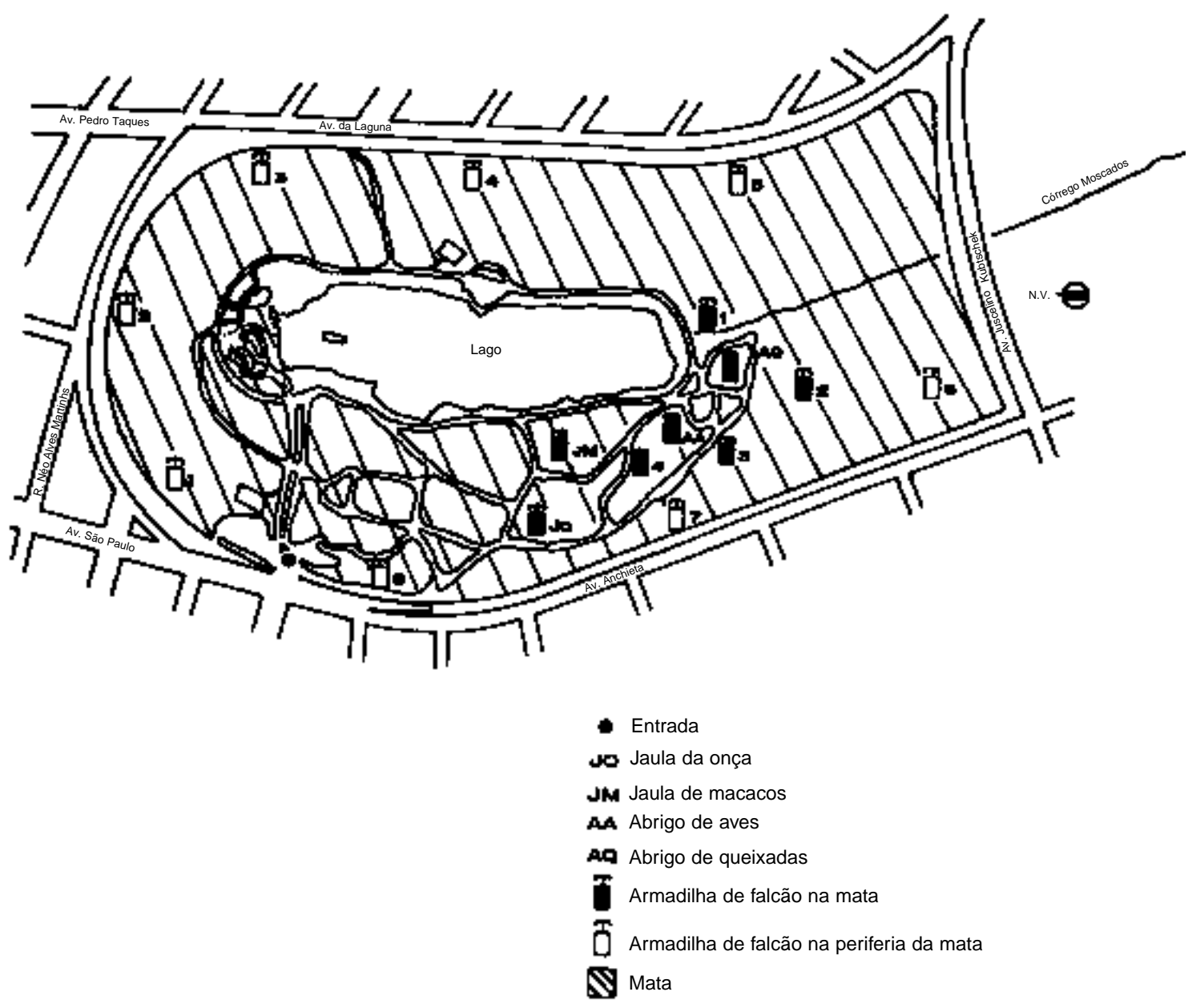

Figura 2 - Distribuição das armadilhas de falcão no Parque do Ingá, no perímetro urbano de Maringá, Paraná, Brasil.

instaladas na periferia da mata.

No Bosque Dois em agosto de 1995 foram instaladas 5 armadilhas dentro da mata em 3 noites consecutivas, das 19 às 22 horas; e mais 6 armadihas, na periferia da mata também em 3 noites consecutivas, em dias diferentes do mês.
No Horto Florestal foram instaladas 6 armadilhas em locais abertos e em viveiros de plantas no interior da mata primitiva modificada que compõe o horto, das 19 às 22 horas, em 3 noites seguidas de setembro de 1995 e; 7 armadilhas nas margens da mata, beirando duas ruas, em 3 noites distintas. 
Apesar dos flebotomíneos terem sido coletados nos mais diversos horários noturnos, especialmente no parque do Ingá, não houve a preocupação de se estabelecer uma metodologia rígida quanto ao horário de coletas, porque o interesse na época em que as coletas foram feitas, era de se fazer um estudo preliminar sobre a fauna e a freqüência de flebotomíneos nas áreas investigadas. Assim, a análise dos dados será feita sem levar em conta o horário de coletas. Por se tratar de um estudo preliminar foi dado maior enfoque aos resultados obtidos no Parque do Ingá. Este local é mais freqüentado como área de lazer, representando área de maior risco de transmissão de Leishmania.

\section{RESULTADOS}

Foram coletados 3.532 flebotomíneos, dos quais $1.527(43,2 \%)$ eram machos e 2.005 (56,8\%) fêmeas.

Na Tabela 1, verifica-se que no Parque do Ingá foram coletados 2.907 flebotomíneos, no Bosque Dois 394 e no Horto Florestal 231, representados por nove, cinco e três espécies, respectivamente. As espécies coletadas foram

Tabela 1 - Flebotomíneos coletados em áreas de matas remanescentes (Parque do Ingá, Bosque Dois e Horto Florestal) e em abrigos de animais silvestres de zoológico, no perímetro urbano de Maringá, Estado do Paraná, Brasil, em 1995.

\begin{tabular}{|c|c|c|c|c|}
\hline \multirow[b]{2}{*}{ Espécie } & \multicolumn{4}{|c|}{ Localidade } \\
\hline & Parque do Ingá & Bosque Dois & Horto Florestal & Total \\
\hline L. whitmani & 2.848 & 376 & 171 & 3.395 \\
\hline L. intermedia & 4 & - & - & 4 \\
\hline L. fischeri & 18 & 6 & 17 & 41 \\
\hline L. pessoai & 4 & - & - & 4 \\
\hline L. migonei & 16 & 2 & 43 & 61 \\
\hline L. firmatoi & 1 & 8 & - & 9 \\
\hline L. monticola & 14 & 2 & - & 16 \\
\hline L. cortelezzii & 1 & - & - & 1 \\
\hline B. brumpti & 1 & - & - & 1 \\
\hline Total & 2.907 & 394 & 231 & 3.532 \\
\hline
\end{tabular}

as seguintes: 1) Brumptomyia brumpti (Larousse, 1920); 2) Lutzomyia cortelezzii (Brèthes, 1924); 3) Lutzomyia firmatoi (Barreto, Martins \& Pellegrino, 1956); 4) Lutzomyia (Pintomyia) fischeri (Pinto, 1926); 5) Lutzomyia (Nyssomyia) intermedia (Lutz \& Neiva, 1912); 6) Lutzomyia migonei (França, 1920); 7) Lutzomyia monticola (Costa Lima, 1932); 8) Lutzomyia (Pintomyia) pessoai (Coutinho \& Barretto, 1940);
9) Lutzomyia (Nyssomyia) whitmani (Antunes \& Coutinho, 1939). Houve prevalência de Lutzomyia whitmani, representando 96,1\% (3.395 exemplares) dos flebotomíneos coletados (3.532). Aliás, esta espécie foi mais freqüente nas três áreas de coletas, representando $98 \%$, (2.848 exemplares) dos flebotomíneos coletados no Parque do Ingá, 95,4\% (376) no Bosque Dois e $74 \%$ (171) no Horto Florestal.

Tabela 2 - Flebotomíneos coletados na mata remanescente e em abrigos de animais silvestres em zoológico do Parque do Ingá, no perímetro urbano de Maringá, Estado do Paraná, Brasil, em 1995.

\begin{tabular}{|c|c|c|c|c|c|c|c|c|c|}
\hline \multirow[b]{2}{*}{ Espécie } & \multicolumn{9}{|c|}{ Armadilha } \\
\hline & AA & $\mathrm{JM}$ & JO & $P Q$ & Subtotal & $\mathrm{MI}$ & $\mathrm{ME}$ & Subtotal & Total \\
\hline L. whitmani & 477 & 142 & 912 & 167 & 1.698 & 1.040 & 110 & 1.150 & 2.848 \\
\hline L. intermedia & - & - & - & - & - & - & 4 & 4 & 4 \\
\hline L. fischeri & 2 & 4 & 3 & 1 & 10 & 6 & 2 & 8 & 18 \\
\hline L. pessoai & - & - & - & - & - & - & 4 & 4 & 4 \\
\hline L. migonei & 2 & - & 7 & 2 & 11 & 4 & 1 & 5 & 16 \\
\hline L. firmatoi & - & - & - & - & - & - & 1 & 1 & 1 \\
\hline L. monticola & 2 & - & 2 & - & 4 & 4 & 6 & 10 & 14 \\
\hline L. cortelezzii & - & - & - & - & - & - & 1 & 1 & 1 \\
\hline B. brumpti & - & - & - & - & - & 1 & - & 1 & 1 \\
\hline Total & 483 & 146 & 924 & 170 & 1.723 & 1.055 & 129 & 1.184 & 2.907 \\
\hline
\end{tabular}

$\mathrm{AA}=$ abrigo de aves; $\mathrm{JM}=$ jaula de macacos; $\mathrm{JO}=$ jaula da onça; $\mathrm{PQ}=$ jaula de queixadas; $\mathrm{MI}=\mathrm{mata}$ interna; $\mathrm{ME}=\mathrm{mata}$ externa. 
$\mathrm{Na}$ Tabela 2 verifica-se que Lutzomyia whitmani prevaleceu em todas as armadilhas instaladas nos abrigos de animais silvestres e na mata no Parque do Ingá. O maior número de flebotomíneos (1.055) capturado no Parque do Ingá ocorreu nas armadilhas instaladas dentro da mata (MI), representando $36,3 \%$ do total coletado (2.907) neste parque. Do total de flebotomíneos coletados nos abrigos de animais silvestres (1.723 exemplares) observou-se que o maior número foi coletado na jaula da onça (924 exemplares) e o menor na jaula de macacos (146 exemplares). No conjunto das armadilhas instaladas às margens externas da mata (ME) do Parque do Ingá coletaram-se 129 flebotomíneos.

\section{DISCUSSÃO}

Os resultados desta pesquisa indicam que a fauna de flebotomíneos tem nove espécies nas matas remanescentes no perímetro urbano de Maringá. Trabalhos anteriores realizados na região norte do Paraná mostraram maior número de espécies de flebotomíneos em matas remanescentes, em domicílios e abrigos de animais domésticos, porém fora do perímetro urbano18 19. Houve prevalência quase que absoluta de L. whitmani. Por se tratar de ambientes parcialmente alterados e circunscritos numa área inteiramente artificial, aqui representada pelo ambiente urbano, pressupõese que poucas espécies de flebotomíneos são capazes de se adaptarem a tal situação, destacando-se $L$. whitmani. A infecção natural deste flebotomíneo por Leishmania tem comprovado o seu potencial vetorial2 12 .

É interessante ressaltar que o número de exemplares de Lutzomyia intermedia coletado foi pequeno, uma vez que esta espécie tem sido assinalada por sua facilidade de adaptação em ambientes com elevado grau antropogênico6. No entanto, Marcondes ${ }^{7}$ afirma que a espécie Lutzomyia intermedia s.l. é constituída "de pelo menos duas espécies distintas, Lutzomyia intermedia s.s. e Lutzomyia neivai". Este autor considera que na região norte do Paraná ocorre a segunda espécie. Analogamente, Rangel et al14 confirmaram a hipótese de que Lutzomyia whitmani s.l. do Estado do Pará é diferente de Lutzomyia whitmani s.s. dos Estados do Ceará e Bahia.

A coleta de maior número de $L$. whitmani no abrigo da onça e das aves, pode ser um sinal da preferência deste flebotomíneo pelo sangue destes animais. Por outro lado, a pequena freqüência desta espécie no abrigo de macacos pode ser um indicador de menor preferência deste díptero pelo sangue de primatas. Tem sido observado que é pequena a freqüência de flebotomíneos dentro de domicílios em áreas rurais no norte do Paraná, quando nas suas proximidades há abrigos de animais domésticos18 19. Todavia, a freqüência de L. whitmani observada neste estudo em todos os abrigos de animais silvestres sugere também seu ecletismo alimentar. Este dado está em sintonia com os poucos trabalhos de análise do conteúdo do tubo intestinal de flebotomíneos da Região Neotropical, que mostram de forma muito clara o ecletismo alimentar destes dípteros 131020.

O presente trabalho mostra melhor adaptação de $L$. whitmani nas matas residuais alteradas e sugere o hematofagismo eclético desta espécie dentro do perímetro urbano de Maringá. Acredita-se contudo que é preciso aprofundar os estudos sobre o comportamento de flebotomíneos nestes ambientes. Cabe ainda investigar qual é a variedade do complexo L. whitmani s.l. que ocorre no Norte do Paraná. Por outro lado, deve ser questionado qual das espécies, $L$. intermedia s.s. ou L. neivai, se adapta melhor nos ambientes antropogênicos, havendo a possibilidade de ambas as espécies se adaptarem nestes ambientes. Por se tratar de uma espécie potencialmente vetora, a predominância quase absoluta de $L$. whitmani torna as áreas investigadas possíveis focos de infecção por Leishmania. Entre as três áreas investigadas o Parque do Ingá oferece maior risco, pois é uma área de lazer freqüentada por grande número de pessoas, onde vivem inúmeros mamíferos silvestres que podem ser reservatórios de Leishmania.

\section{AGRADECIMENTOS}

Ao Sr. Carmelindo Colato, desenhista e projetista do Departamento de Engenharia Civil da Universidade Estadual de Maringá, pelos serviços de desenhista.

\section{REFERÊNCIAS BIBLIOGRÁFICAS}

1. Alexander B, Ferro C, Young DG, Morales A, Tesh RB. Ecology of sand flies (Diptera:Psychodidae) in a focus of Leishmania (Viannia) braziliensis in Northeastern Colombia. Memórias do Instituto Oswaldo Cruz 87:387-395, 1992. 
2. Azevedo ACR, Rangel EF, Costa EM, David J, Vasconcelos AW, Lopes UG. Natural infection of L. (Nyssomyia) whitmani (Antunes \& Coutinho,1939) by Leishmania of the braziliensis complex in Baturité, Ceará State, Northeastern BraziL. Memórias do Instituto Oswaldo Cruz 85:251, 1990.

3. Christensen HA, Vasquez AM. The tree-buttress biotipe: A pathobiocenose of Leishmania braziliensis. The American Journal of Tropical Medicine and Hygiene 31:243-251, 1982.

4. Falcão AR. Um novo modelo de armadilha luminosa de sucção para pequenos insetos. Memórias do Instituto Oswaldo Cruz 76:303-305, 1981.

5. Falqueto A, Sessa PA, Varejão JBM, Ferreira AL. Leishmaniose tegumentar (LT) na região metropolitana de Vitória, Espírito Santo. In: Resumos do XXXI Congresso da Sociedade Brasileira de Medicina Tropical, São Paulo, p. 131, 1995.

6. Gomes AC, Santos JLF, Galati EAB. Ecological aspectos of american cutaneous leishmaniasis. 4. Observations on the endophilic behavior of the sandfly and vectorial role of Psychodopygus intermedius in the Ribeira Valley of the São Paulo State, Brazil. Revista de Saúde Pública 20:280-287, 1986.

7. Marcondes CB. Morfometria e DNA mitocondrial de populações sulamericanas de Lutzomyia (Nyssomyia) intermedia (Lutz \& Neiva, 1912) (Diptera, Psychodiade, Phlebotominae). Tese. Universidade Federal do Paraná, Curitiba, 1997.

8. Ministério da Saúde (Fundação Nacional de Saúde. Casos notificados de leishmaniose tegumentar americana. Residentes na UF, por ano, segundo Unidade Federada. Brasil, Brasil - 1980 a 1994. s.l.: s.n., Mimeografado. 1995.

9. Ministério da Saúde (Fundação Nacional de Saúde. Número de casos notificados de leishmaniose tegumentar americana. Residentes na UF, segundo Unidade Federada, Brasil - 1994. s.l.: s.n., Mimeografado. 1996.

10. Morrison AC, Ferro C, Tesh RB. Host preferences of sand fly $L$. longipalpis at an endemic focus of american visceral leishmaniasis in Colombia. The American Journal of Tropical Medicine and Hygiene 49:68-75, 1993.

11. Pereira GFM, Fonseca HHR. Leishmaniose tegumentar americana: epidemiologia e controle. Revista da Sociedade Brasileira de Medicina Tropical 27(supl III):45-50, 1994.
12. Queiroz RG, Vasconcelos IAB, Vasconcelos AW, Pessoa FAC, Sousa RN, David JR. Cutaneous leishmaniasis in Ceara in Northeastern Brazil: incrimination of Lutzomyia whitmani (Diptera: Psychodidae) as a vector of Leishmania braziliensis in Baturité maunicipality. The American Journal of Tropical Medicine and Hygiene 50:693-698, 1994.

13. Rangel EF, Azevedo ACR, Andrade CA, Souza NA, Wermelinger ED. Studies on sandfly fauna (Diptera: Psychodidae) in a foci of cutaneous leishmaniasis in Mesquita, Rio de Janeiro State, BraziL. Memórias do Instituto Oswaldo Cruz 85:39-45, 1990.

14. Rangel EF, Lainson R, Souza AA, Ready P, Azevedo ACR. Variation between geographical populatins of Lutzomyia (Nyssomyia) whitmani (Antunes \& Coutinho, 1939) sensu latu (Diptera: Psychodidae: Phlebotominae) in Brazil. Memórias do Instituto Oswaldo Cruz 91:43-50, 1996.

15. Roberto ACBS, Lima AP, Peixoto PR; Misuta NM, Fukushigue $Y$, Ferreira MEMC, Nerilo Sobrinho A, Silveira TGV e Teodoro U. Avaliação da terapia com antimoniato de N-Metil glucamina e de notificação de leishmaniose tegumentar. Anais brasileiros de Dermatologia 72:129-136, 1997.

16. Sucam - Ministério da Saúde. Leishmaniose tegumentar americana no Brasil: sua problemática, seu controle, desafios e perspectivas. Informações Epidemiolócicas. Superintendência de Campanhas 5: 1987.

17. Stolf HO, Marques SA, Marques MEA, Yoshida ELA, Dillon NL. Surto de leishmaniose tegumentar americana em Itaporanga, São Paulo (Brasil). Revista do Instituto de Medicina Tropical de São Paulo 35:437-442, 1993.

18. Teodoro U. Características ecológicas de flebotomíneos (Diptera, Psychodidae) em habitats antrópicos, município de Jussara, Paraná, Brasil. Tese de Doutorado. Universidade Federal do Paraná, Curitiba, 1995.

19. Teodoro U, La Salvia Fo V, Lima EM, Spinosa RP, Barbosa OC, Ferreira MEMC, Lonardoni, MVC. Observações sobre o comportamento de flebotomíneos em ecótopos florestais e extraflorestais, em área endêmica de leishmaniose tegumentar americana, no norte do Estado do Paraná, sul do BrasiL. Revista de Saúde Pública 27:242-249, 1993.

20. Tesh RB Chaniotis BN, Carrera BR, Johnson KM. Further studies on the natural preferences of panamanian phlebotomine sandflies. American 